\title{
Keck Planet Imager and Characterizer (KPIC): status update
}

D. Mawet, C. Z. Bond, J.-R. Delorme, N. Jovanovic, S. Cetre, et al.

D. Mawet, C. Z. Bond, J.-R. Delorme, N. Jovanovic, S. Cetre, M. Chun, D. Echeverri, D. Hall, S. Lilley, J. K. Wallace, P. Wizinowich, "Keck Planet Imager and Characterizer (KPIC): status update," Proc. SPIE 10703, Adaptive Optics Systems VI, 1070306 (10 July 2018); doi: 10.1117/12.2314037

Event: SPIE Astronomical Telescopes + Instrumentation, 2018, Austin, Texas, United States 


\title{
Keck Planet Imager and Characterizer: status update
}

\author{
D. Mawet ${ }^{a, b}$, C. Z. Bond ${ }^{c}$, J.-R. Delorme ${ }^{a}$, N. Jovanovic ${ }^{a}$, S. Cetre ${ }^{d}$, M. Chun ${ }^{c}$, D. Echeverri ${ }^{a}$, \\ D. Hall ${ }^{c}$, S. Lilley ${ }^{d}$, J.K. Wallace ${ }^{b}$, and P. Wizinowich ${ }^{d}$ \\ ${ }^{a}$ Department of Astronomy, California Institute of Technology, Pasadena, CA 91106, USA; \\ ${ }^{b}$ Jet Propulsion Laboratory, California Institute of Technology, Pasadena, CA 91109, USA; \\ ${ }^{c}$ W. M. Keck Observatory, 65-1120 Mamalahoa Hwy., Kamuela, HI, USA 96743; \\ ${ }^{d}$ Institute for Astronomy, University of Hawaii, 640 N. Aóhoku Place, Hilo, HI, USA 96720;
}

\begin{abstract}
Here we report on the status of the The Keck Planet Imager and Characterizer (KPIC), which is an on-going series of upgrades to the W.M. Keck II adaptive optics system and instrument suite focused on exoplanet imaging and spectroscopic characterization. The KPIC infrared pyramid wavefront sensor and fiber injection unit to highresolution infrared spectrograph NIRSPEC have been assembled, integrated and are under-going tests at the University of Hawaii before installation at the Summit in the Fall of 2018.
\end{abstract}

Keywords: Exoplanets, high contrast imaging, high contrast high resolution spectroscopy, small inner working angle coronagraphy, vortex coronagraph, on-axis segmented telescopes, apodization, Extremely Large Telescopes

\section{INTRODUCTION}

The Keck Planet Imager and Characterizer (KPIC) consists of an on-going series of upgrades to the W.M. Keck II adaptive optics system, facility infrared imager NIRC2 and high-resolution spectrograph NIRSPEC. The upgrades aim to enable unique exoplanet science. NIRC2 was upgraded with an L-band vortex coronagraph, ${ }^{1-5}$ now offered in shared-risk mode. The NIRC2 L-band vortex enabled the discovery of new protoplanet candidates, ${ }^{6,7}$ disks, ${ }^{8}$ and allowed us to constraint mass accretion rates in protoplanetary disks with gaps. ${ }^{9}$

\section{KPIC SCIENCE CASES}

Exoplanets around late-type stars. M dwarfs constitute a promising reservoir to survey in order to advance our understanding of planetary formation and evolution. Indeed, $\mathrm{M}$ dwarfs outnumber all earlier-type stars together. ${ }^{10}$ Their abundance, low close binary fraction, and the ubiquitous presence of massive protoplanetary disks at young ages imply that they are common sites of planet formation. Close separations $(<1 \mathrm{AU})$ have been extensively probed by Doppler and transit surveys with the following results: the frequency of close-in giant planets $\left(1-10 M_{J u p}\right)$ is only $2.5 \pm 0.9 \%$, consistent with core accretion plus migration models. ${ }^{11}$ On the other hand, the Kepler survey indicates that Earth- to Neptune-sized planets might be as common as one per star. ${ }^{12-14}$ The outskirts of young M-dwarf systems (10 - $\left.100 \mathrm{AU}\right)$ have been probed by first-generation direct imaging instruments, and results show that massive planets are rare: fewer than $10.6 \%$ of M-dwarf systems surveyed harbor $1-13 M_{\text {Jup }}$ giant planets in their outer regions. ${ }^{15}$ Disk instability does not seem to be a common mechanism of giant planet formation. The 1-10 AU parameter space is thus believed to be the overwhelmingly favored region for planet formation. Across the entire range of sensitivity $\left(10 M_{\oplus}-10 M_{J u p}\right)$, the occurrence rates measured by microlensing surveys imply an average $1.6_{-0.9}^{+0.7}$ planets per star ${ }^{16}$ ! Microlensing probes the full range of planetary masses in this region, but the masses and metallicities of the host stars are usually poorly constrained and so are of limited value for statistical studies. Moreover, one-shot microlensingdetected objects can not be followed up. High contrast imaging with a good knowledge of the host star distance and age is therefore the perfect complement to indirect techniques, and holds the promise of filling in this

Further author information: send correspondence to dmawet@astro.caltech.edu 
untouched parameter space, and provide excellent characterization opportunities. Last but not least, M dwarfs provide the best star-planet contrast ratios for young giant planets among all stellar masses.

KPIC will target this reservoir with the most advanced high contrast imaging technologies, unveiling these planets with unprecedented sensitivity, but also directly analyzing their thermal light with high-resolution spectroscopy (NIRSPEC), enabling the retrieval of the molecular composition of their atmosphere ${ }^{17}$ and in favorable cases the Doppler imaging of cloud, wind, and temperature gradients. ${ }^{18-20}$

Planetary systems in star forming regions (SFR). Sky coverage with an infrared WFS is typically $50 \%$ higher than with a classical visible WFS. ${ }^{21}$ In obscured areas such as SFR, the gain is much more dramatic. The population of young stars in Taurus, $140 \mathrm{pc}$ away, is dominated by $\mathrm{M}$ stars and very late $\mathrm{K}$ stars ${ }^{22}$ making infrared WFS essential for these very red stars. Indeed, an R-band WFS sensitivity rolloff at $R \simeq 13$ currently provides access to only a handful of $\mathrm{T}$ Tauri stars, while a rolloff at $J / H \simeq 13$ mags would enable high contrast on a hundred young stars in Taurus alone. Thus, infrared wavefront sensing enables high contrast imaging studies of extrasolar planetary systems (both disk + protoplanets) in their infancy.

High Dispersion Coronagraphy to characterize young giant planets. Coupling a high-resolution spectrograph with a high-contrast imaging instrument (Fig. 1) is the next big step in the direct characterization of exoplanet atmospheres. ${ }^{20}$ In this scheme, the high-contrast imaging system serves as a spatial filter to separate the light from the star and the planet, and the high-resolution spectrograph serves as spectral filter, which differentiates between features in the stellar and planetary spectra, e.g., between different absorption lines and radial velocities. High-resolution spectroscopy has three game-changing benefits:

1. Detailed species-by-species molecular characterization (spectral retrieval).

2. Doppler measurements (planet spin, orbital velocity, plus mapping of atmospheric and/or surface features).

3. Last but not least, improved detection capability by side-stepping speckle noise calibration issues affecting low-resolution spectroscopic data from current integral field spectrographs such as SPHERE/IFS, SCExAO/CHARIS, and GPI.

Most direct imaging results so far have indeed focused on photometry and very low-resolution spectroscopy, due to the lack of instruments designed to optimally merge high contrast imaging (wavefront control and coronagraphy) and high-resolution spectroscopy at small angles from the host star. ${ }^{23,24}$ KPIC, and its fiber injection unit (FIU) linking Keck AO to NIRSPEC would enable routine high-resolution characterization of directly imaged low-mass companions and exoplanets (see Table 1). The KPIC FIU to NIRSPEC will also make Doppler imaging mainstream. ${ }^{18}$

Characterization of close-in planets. For transiting and non-transiting hot Jupiters and warm Neptunes, high-resolution transmission spectroscopy has been used to detect molecular gas ${ }^{25-27}$ and to study day to night side wind velocity, ${ }^{25}$ providing an ultimate test for $3 \mathrm{D}$ exoplanet atmosphere models. For planets detected with the RV method, spectral lines owing to the planet and star may be separated via their radial velocities $(>50$ $\mathrm{km} / \mathrm{s}$ ). The RV of a planet can thus be measured to break the degeneracy between the true planet mass and orbital inclination intrinsic to RV detections. ${ }^{28}$ Together with NIRSPEC, KPIC will provide the sensitivity, spectral resolution, and spectral coverage necessary to pursue such studies, on time for high scientific value and unique follow-up opportunities in the TESS era.

Protoplanetary disks. Dust and gas accretion disks around young stars form as a natural consequence of the conservation of angular momentum in a collapsing cloud core. Planets are born in their dense dusty midplane disk, called protoplanetary (PP) disks. The evolution, and ultimately the dispersion of PP disks, holds the key to many open questions related to planet formation. High-resolution infrared spectroscopy is instrumental in understanding PP disk gas dynamics through, e.g. the study of the emission and absorption of CO ro-vibrational 


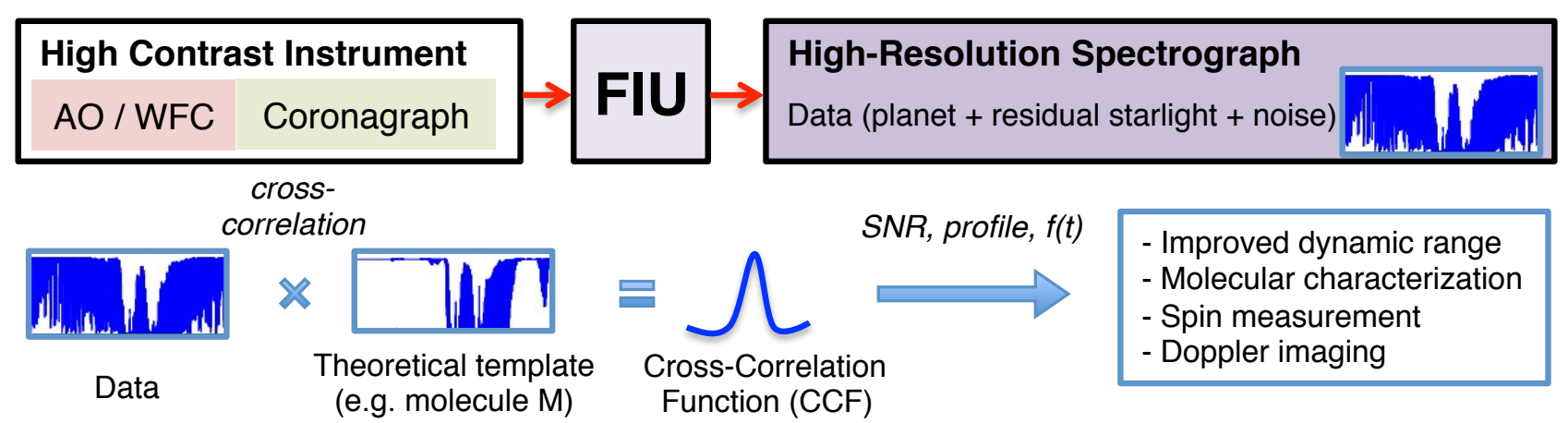

Figure 1. High-dispersion coronagraphy (HDC) concept. A classical high-contrast instrument, with an adaptive optics (AO) or wavefront control (WFC) system followed by a starlight suppressing coronagraph, is linked to a high-resolution spectrograph uby a fiber injection unit (FIU). The data processing steps are as follows: the raw data (planet+residual starlight unfiltered by the high-contrast instrument, and various noise contributors such as photon shot noise, readout noise, background noise, etc.) is cross-correlated with a theoretical template yielding a new observable, called here the cross-correlation function $(\mathrm{CCF})$. The $\mathrm{CCF}$ profile provides improved dynamic range for detection and molecular characterization. Its broadening with respect to the instrument line profile is a direct measure of the planet's spin rotation. The variation of the line profile morphology over the rotation period enables Doppler imaging.

\begin{tabular}{|c|c|c|c|c|c|c|c|c|c|c|c|c|}
\hline Host name & Pl. & Sep (AU) & Sep (") & $\mathrm{M}_{J}$ & RA & DEC & $\mathrm{d}(\mathrm{pc})$ & SpTy & $\mathrm{V}$ & $\mathrm{H}$ & $\mathrm{K}$ & $\mathrm{L}$ \\
\hline \multicolumn{13}{|l|}{ Below DBL } \\
\hline $51 \mathrm{Eri}$ & $\mathrm{b}$ & 13.2 & 0.4 & 2 & $04 \mathrm{~h} 37 \mathrm{~m} 36.13 \mathrm{~s}$ & $-02 \mathrm{~d} 28 \mathrm{~m} 24.8 \mathrm{~s}$ & 29 & Fo IV & 5.2 & 4.8 & 4.5 & 4.5 \\
\hline 2MASS J1207 & $\mathrm{b}$ & 46 & 0.9 & 4 & $12 \mathrm{~h} 07 \mathrm{~m} 33.47 \mathrm{~s}$ & $-39 \mathrm{~d} 32 \mathrm{~m} 54.0 \mathrm{~s}$ & 52 & M8 & 20.2 & 12.4 & 11.9 & 11.6 \\
\hline GJ 504 & b & 43.5 & 2.4 & 4 & $13 \mathrm{~h} 16 \mathrm{~m} 46.52 \mathrm{~s}$ & $+09 \mathrm{~d} 25 \mathrm{~m} 27.0 \mathrm{~s}$ & 18 & Go V & 5.2 & 4.1 & 4.0 & 3.8 \\
\hline Ross 458 & c & 1168 & 102.0 & 6 & $13 \mathrm{~h} 00 \mathrm{~m} 46.58 \mathrm{~s}$ & $+12 \mathrm{~d} 22 \mathrm{~m} 32.6 \mathrm{~s}$ & 11 & M0.5 & 9.7 & 5.8 & 5.6 & 5.5 \\
\hline HR 8799 & $\mathrm{~b}$ & 68 & 1.7 & 7 & $23 \mathrm{~h} 07 \mathrm{~m} 28.72 \mathrm{~s}$ & $+21 \mathrm{~d} 08 \mathrm{m03} .3 \mathrm{~s}$ & 39 & $\mathrm{FO}+\mathrm{V}$ & 6.0 & 5.3 & 5.2 & 5.2 \\
\hline 2MASS J0441 & $\mathrm{b}$ & 15 & 0.1 & 8 & $04 \mathrm{~h} 41 \mathrm{~m} 44.90 \mathrm{~s}$ & $+23 \mathrm{~d} 01 \mathrm{~m} 51.4 \mathrm{~s}$ & 140 & M8.5 & & 13.7 & 13.2 & \\
\hline 1RXS J1609 & $\mathrm{b}$ & 330 & 2.3 & 8 & $16 \mathrm{~h} 09 \mathrm{~m} 30.30 \mathrm{~s}$ & $-21 \mathrm{~d} 04 \mathrm{~m} 58.9 \mathrm{~s}$ & 145 & K7 V & & 9.1 & 8.9 & 8.8 \\
\hline HIP 65426 & b & 92 & 0.8 & 9 & $13 \mathrm{~h} 24 \mathrm{~m} 36.10 \mathrm{~s}$ & $-51 \mathrm{~d} 30 \mathrm{~m} 16.1 \mathrm{~s}$ & 111 & A2 $\mathrm{V}$ & 7.0 & 6.9 & 6.8 & 6.8 \\
\hline ROXs $42 \mathrm{~B}$ & $\mathrm{~b}$ & 157 & 1.2 & 9 & $16 \mathrm{~h} 31 \mathrm{~m} 15.02 \mathrm{~s}$ & $-24 \mathrm{~d} 32 \mathrm{~m} 43.7 \mathrm{~s}$ & 135 & Mo & & 9.0 & 8.7 & 8.5 \\
\hline HR 8799 & c & 38 & 1.0 & 10 & $23 \mathrm{~h} 07 \mathrm{~m} 28.72 \mathrm{~s}$ & $+21 \mathrm{~d} 08 \mathrm{~m} 03.3 \mathrm{~s}$ & 39 & $\mathrm{FO}+\mathrm{V}$ & 6.0 & 5.3 & 5.2 & 5.2 \\
\hline HR 8799 & d & 24 & 0.6 & 10 & $23 \mathrm{~h} 07 \mathrm{~m} 28.72 \mathrm{~s}$ & $+21 \mathrm{~d} 08 \mathrm{~m} 03.3 \mathrm{~s}$ & 39 & $\mathrm{FO}+\mathrm{V}$ & 6.0 & 5.3 & 5.2 & 5.2 \\
\hline HR 8799 & e & 14.5 & 0.4 & 10 & $23 \mathrm{~h} 07 \mathrm{~m} 28.72 \mathrm{~s}$ & $+21 \mathrm{~d} 08 \mathrm{~m} 03.3 \mathrm{~s}$ & 39 & $\mathrm{FO}+\mathrm{V}$ & 6.0 & 5.3 & 5.2 & 5.2 \\
\hline PDS 70 & b & 22 & 0.2 & 10 & $14 \mathrm{~h} 08 \mathrm{~m} 10.15 \mathrm{~s}$ & $-41 \mathrm{~d} 23 \mathrm{~m} 52.57 \mathrm{~s}$ & 113 & K5 & 12.2 & 8.8 & 8.5 & 7.9 \\
\hline CFBDSIR J1458 & $\mathrm{b}$ & 2.6 & 0.1 & 11 & $14 \mathrm{~h} 58 \mathrm{~m} 29.00 \mathrm{~s}$ & $+10 \mathrm{~d} 13 \mathrm{~m} 43.0 \mathrm{~s}$ & 23 & & & & & \\
\hline DH Tau & $\mathrm{b}$ & 330 & 2.4 & 11 & $04 \mathrm{~h} 29 \mathrm{~m} 41.56 \mathrm{~s}$ & $+26 \mathrm{~d} 32 \mathrm{~m} 58.3 \mathrm{~s}$ & 140 & M0.5 & & 8.8 & 8.2 & 7.4 \\
\hline VHS J1256 & $\mathrm{b}$ & 102 & 8.0 & 11 & $12 \mathrm{~h} 56 \mathrm{m02} .15 \mathrm{~s}$ & $-12 \mathrm{~d} 57 \mathrm{~m} 21.7 \mathrm{~s}$ & 13 & M7.5 & 17.8 & 10.5 & 10.0 & 9.9 \\
\hline GU Psc & $\mathrm{b}$ & 2000 & 41.7 & 11 & $01 \mathrm{~h} 12 \mathrm{~m} 35.04 \mathrm{~s}$ & $+17 \mathrm{~d} 03 \mathrm{~m} 55.7 \mathrm{~s}$ & 48 & M3 & 13.6 & 9.6 & 9.3 & 9.3 \\
\hline 2MASS J2236 & $\mathrm{b}$ & 230 & 3.1 & 13 & $22 \mathrm{~h} 36 \mathrm{~m} 24.53 \mathrm{~s}$ & $+47 \mathrm{~d} 51 \mathrm{~m} 42.6 \mathrm{~s}$ & 74 & $\mathrm{~K} 7 \mathrm{~V}$ & 12.5 & 9.3 & 9.1 & 9.1 \\
\hline bet Pic & $\mathrm{b}$ & 9.1 & 0.5 & 13 & $05 \mathrm{~h} 47 \mathrm{~m} 17.09 \mathrm{~s}$ & $-51 \mathrm{~d} 03 \mathrm{~m} 59.4 \mathrm{~s}$ & 19 & A5 V & 3.9 & 3.5 & 3.5 & 3.5 \\
\hline \multicolumn{13}{|l|}{ Above DBL } \\
\hline SR 12 AB & $\mathrm{c}$ & & 0.0 & 13 & $16 \mathrm{~h} 27 \mathrm{~m} 19.51 \mathrm{~s}$ & $-24 \mathrm{~d} 41 \mathrm{~m} 40.4 \mathrm{~s}$ & & & 13.3 & 8.6 & 8.4 & 8.3 \\
\hline kap And & $\mathrm{b}$ & 55 & 1.1 & 14 & $23 \mathrm{~h} 40 \mathrm{~m} 24.51 \mathrm{~s}$ & $+44 \mathrm{~d} 20 \mathrm{m0} 2.2 \mathrm{~s}$ & 52 & B9 IV & 4.1 & 4.6 & 4.6 & 4.3 \\
\hline 2MASS J0219 & $\mathrm{b}$ & 156 & 4.0 & 14 & $02 \mathrm{~h} 19 \mathrm{~m} 22.11 \mathrm{~s}$ & $-39 \mathrm{~d} 25 \mathrm{~m} 22.5 \mathrm{~s}$ & 39 & M6 & 15.2 & 10.8 & 10.4 & 10.1 \\
\hline Oph 11 & $\mathrm{~b}$ & 243 & 1.9 & 14 & $16 \mathrm{~h} 22 \mathrm{~m} 25.21 \mathrm{~s}$ & $-24 \mathrm{~d} 05 \mathrm{~m} 13.9 \mathrm{~s}$ & 125 & M9 & & 13.8 & 13.4 & 12.8 \\
\hline USco CTIO 108 & $\mathrm{~b}$ & 670 & 0.0 & 14 & $16 \mathrm{~h} 05 \mathrm{~m} 54.08 \mathrm{~s}$ & $-18 \mathrm{~d} 18 \mathrm{~m} 44.4 \mathrm{~s}$ & & M7 & & 12.8 & 12.5 & 12.1 \\
\hline ROXs 12 & b & 210 & 1.8 & 16 & $16 \mathrm{~h} 26 \mathrm{~m} 27.75 \mathrm{~s}$ & $-25 \mathrm{~d} 27 \mathrm{~m} 24.7 \mathrm{~s}$ & 120 & Mo & 15.6 & 9.9 & 9.2 & 8.4 \\
\hline GSC 06214 & b & 320 & 2.2 & 16 & $16 \mathrm{~h} 21 \mathrm{~m} 54.67 \mathrm{~s}$ & $-20 \mathrm{~d} 43 \mathrm{~m} 09.1 \mathrm{~s}$ & 145 & $\mathrm{~K} 7$ & 12.4 & 9.3 & 9.2 & 9.1 \\
\hline FU Tau & $\mathrm{b}$ & 800 & 5.7 & 16 & $04 \mathrm{~h} 23 \mathrm{~m} 35.74 \mathrm{~s}$ & $+25 \mathrm{~d} 02 \mathrm{~m} 59.6 \mathrm{~s}$ & 140 & M7.25 & & 13.9 & 13.3 & \\
\hline GQ Lup & b & 100 & 0.7 & 20 & $15 \mathrm{~h} 49 \mathrm{~m} 12.10 \mathrm{~s}$ & $-35 \mathrm{~d} 39 \mathrm{~m} 05.1 \mathrm{~s}$ & 139 & K7e V & 12.4 & 7.7 & 7.1 & 6.1 \\
\hline $2 \mathrm{MASS} J 2140$ A & b & & 0.0 & 21 & $21 \mathrm{~h} 40 \mathrm{~m} 29.32 \mathrm{~s}$ & $+16 \mathrm{~d} 25 \mathrm{~m} 18.3 \mathrm{~s}$ & 25 & M8.5 & & 12.3 & 11.8 & 11.4 \\
\hline HN Peg & b & 773 & 42.0 & 22 & $21 \mathrm{~h} 44 \mathrm{~m} 31.33 \mathrm{~s}$ & $+14 \mathrm{~d} 46 \mathrm{~m} 19.0 \mathrm{~s}$ & 18 & Go IV-V & 6.0 & 4.6 & 4.6 & 4.5 \\
\hline WISEP J12172 A & b & 8 & 0.8 & 22 & $12 \mathrm{~h} 17 \mathrm{~m} 56.91 \mathrm{~s}$ & $+16 \mathrm{~d} 26 \mathrm{~m} 40.2 \mathrm{~s}$ & 10 & $\mathrm{~T} 8.5$ & & & & 16.5 \\
\hline HIP 78530 & $\mathrm{~b}$ & 740 & 4.7 & 23 & $16 \mathrm{~h} 01 \mathrm{~m} 55.46 \mathrm{~s}$ & $-21 \mathrm{~d} 58 \mathrm{~m} 49.4 \mathrm{~s}$ & 157 & B9 V & 7.2 & 6.9 & 6.9 & 6.9 \\
\hline HD 203030 & $\mathrm{~b}$ & 487.1 & 11.9 & 24 & $21 \mathrm{~h} 18 \mathrm{~m} 58.22 \mathrm{~s}$ & $+26 \mathrm{~d} 13 \mathrm{~m} 50.0 \mathrm{~s}$ & 41 & G8 V & 8.4 & 6.7 & 6.7 & 6.7 \\
\hline 2MASS J0122 & $\mathrm{b}$ & 52 & 1.4 & 25 & $01 \mathrm{~h} 22 \mathrm{~m} 50.94 \mathrm{~s}$ & $-24 \mathrm{~d} 39 \mathrm{~m} 50.6 \mathrm{~s}$ & 36 & $\mathrm{M} 3.5 \mathrm{~V}$ & 14.2 & 9.5 & 9.2 & 9.0 \\
\hline Fomalhaut & b & 160 & 20.8 & $?$ & $22 \mathrm{~h} 57 \mathrm{~m} 39.05 \mathrm{~s}$ & $-29 \mathrm{~d} 37 \mathrm{~m} 20.1 \mathrm{~s}$ & 8 & A3 V & 1.2 & 0.9 & 0.9 & -1.5 \\
\hline $\mathrm{LkCa} 15$ & b & 14.7 & 0.0 & ? & $04 \mathrm{~h} 39 \mathrm{~m} 17.80 \mathrm{~s}$ & $+22 \mathrm{~d} 21 \mathrm{~m} 03.5 \mathrm{~s}$ & & & 12.0 & 8.6 & 8.2 & 7.5 \\
\hline LkCa 15 & c & 18.6 & 0.0 & ? & $04 \mathrm{~h} 39 \mathrm{~m} 17.80 \mathrm{~s}$ & $+22 \mathrm{~d} 21 \mathrm{~m} 03.5 \mathrm{~s}$ & & & 12.0 & 8.6 & 8.2 & 7.5 \\
\hline MWC 758 & b & 17 & 0.11 & ? & $05 \mathrm{~h} 30 \mathrm{~m} 27.53 \mathrm{~s}$ & $+25 \mathrm{~d} 19 \mathrm{~m} 57.08 \mathrm{~s}$ & 151 & A $8 \mathrm{~V}$ & 8.5 & 6.5 & 5.8 & 4.75 \\
\hline
\end{tabular}

Table 1. Target list for HDC with KPIC.

lines. Spatially resolved high-resolution spectro-astrometry (at mas scale) of the molecular gas allows measuring its distribution in space and velocity, correlate it to disk geometries and accretion activity. Infrared spectroscopy also enables detailed characterization of the molecular composition of young disk, including organic molecules.

Detecting and characterizing young forming planets. Answering the key question How do giant planets from? can not be achieved by only observing older, dynamically evolved systems. Detecting extrasolar giant planets near the epoch of their formation is much easier, due to their higher luminosities. This requires the full angular resolution of Keck, in order to separate giant planets at solar system scales from their host stars. Most of the luminosity of these forming planets is expected to be emitted at near-infrared wavelengths ${ }^{29}$ from a circumplanetary disk. It has so far proved difficult to uniquely separate circumplanetary emission from circumstellar 


\begin{tabular}{|l|l|}
\hline AO upgrades & \\
\hline Sensing architecture & Modulated Pyramid \\
Detector & Leonardo SAPHIRA \\
Readout noise & $<1 e^{-}$ \\
Sensing wavelength & $\mathrm{H}$ band (J band optional) \\
Temporal bandwidth & $1.5 \mathrm{kHz}$ \\
Deformable mirror & $3.5 \mu \mathrm{m} 34 \mathrm{x} 34$ BMC MEMS DM \\
\hline Imaging & NIRC2 \\
\hline Wavelength & 1 to 5 microns (Y to M) \\
Angular resolution & 20 to 100 mas \\
Coronagraph & L-band vector vortex \\
Inner working angle & $0 " .1$ \\
Contrast after processing & $10^{-5}$ at $0 " .5$ \\
\hline Spectroscopy & NIRSPEC \\
\hline Wavelength & 2 to 4 microns (K and L) \\
Angular resolution & 40 to 100 mas \\
Inner working angle & 40 to 100 mas \\
Fiber input & 5 science single-mode fibers \\
Fiber patrol radius from the star & $\sim 2.5 "$ \\
Spectral resolution & 37,500 \\
Contrast with HDC & $10^{-6}$ at 0".5 \\
\hline
\end{tabular}

Table 2. KPIC specifications.

disk emission, as this requires resolving line emission at high spectral dispersion. The kinematic signatures of these warm disks will occur in the range of $3-30 \mathrm{~km} / \mathrm{s}$ (similar to the velocities of Jupiters moons), with strong line emission from $\mathrm{CO}$ and $\mathrm{H}_{2} \mathrm{O}$ in the bandpass of HISPEC. These data might even lead to measurements of the dynamical masses of these forming exoplanets, in much the same way as one can derive masses of $\mathrm{T}$ Tauri stars from the velocity curves of circumstellar disks. Finally, Doppler monitoring of directly-imaged planets could lead to discoveries of exomoons. ${ }^{30}$

\section{KPIC CAPABILITIES}

KPIC is both capable of taking images of exoplanets with Keck II infrared imager NIRC2 and perform highresolution spectroscopic characterization of their atmospheres with NIRSPEC. Table 2 summarizes KPIC's specifications.

\section{KPIC STATUS BY SUBSYSTEM}

In this section we present a brief overview of the status of KPIC's subsystems integration. A block diagram is shown in Figure 2.

\subsection{NIRC2 coronagraph}

The NIRC2 L-band vortex coronagraph was commissioned in Spring/Summer 2015 and is now available in shared-risk mode. ${ }^{1-5}$

\subsection{Infrared Pyramid WFS}

The infrared Pyramid Wavefront sensor of KPIC is described in detail in Bond et al. 2018 (these proceedings). The system was recently integrated with an infrared SAPHIRA e-APD array and successfully demonstrated $<1 e^{-}$readout noise at high gain at the University of Hawaii (Hilo). The sensor was paired to the fiber injection unit (Figure 3) and will soon be installed on the Keck II AO bench (Fall of 2018).

More details about the opto-mechanical design of the sensor can be found in Lilley et al. 2018 (these proceedings). The real-time control system of KPIC is described in Cetre et al. 2018 (these proceedings). 

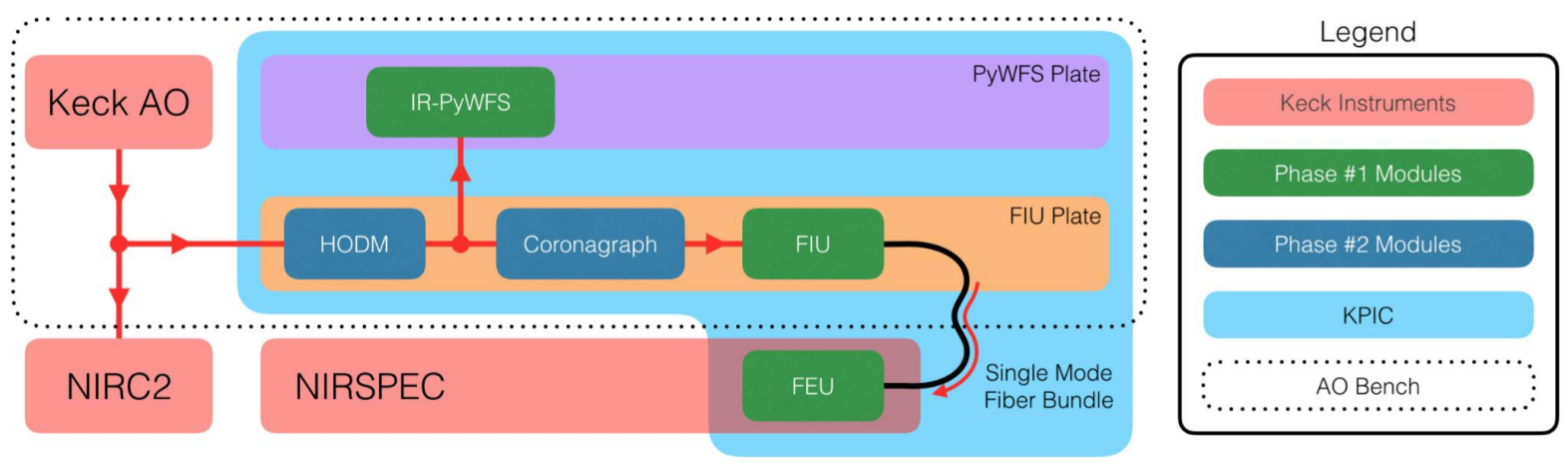

Figure 2. Block diagram of KPIC showing the different subsystems and the links between them. HODM - High Order DM, FIU - Fiber Injection Unit, IR-PyWFS - infrared Pyramid Wavefront Sensor, FEU - Fiber Extraction Unit.
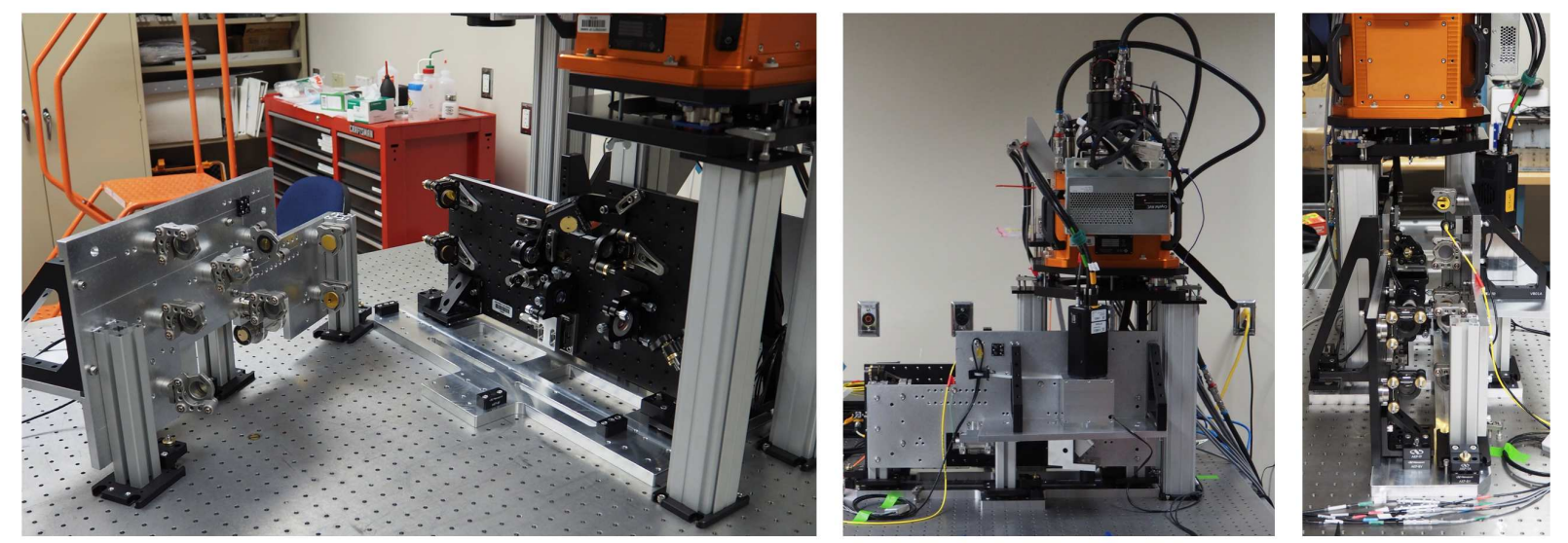

Figure 3. Left: FIU plate (silver) in front of the PyWFS plate (Black). Middle: picture of KPIC from the back side of the FIU plate. Right: Picture of the space between the two plates when co-aligned.

\subsection{FIU and FEU to NIRSPEC}

The fiber injection unit linking the Keck II AO bench to NIRSPEC is discussed in details in Delorme et al. 2018 (these proceedings). The system was integrated and paired to the infrared Pyramid WFS (Figure 3), and will be installed at the Summit in the Fall of 2018. The fiber extraction unit will be integrated to the NIRSPEC calibration unit this Summer (2018).

\section{CONCLUSIONS AND PERSPECTIVES}

KPIC, with its IWA of $\simeq 40$ mas at K-band and $\simeq 80$ mas at L-band, will complement JWST at very small angles (the IWA of NIRCAM and MIRI will be 5-10 times larger). KPIC's high resolution spectroscopic mode will demonstrate crucial capabilities that will be essential to follow-up discoveries from JWST, TESS and later on WFIRST CGI. KPIC will bridge the gap between the Keck AO system and a TMT planetary systems imager (PSI). Keck provides a pathfinder for ExAO and high dispersion coronagraphy on the highly-segmented TMT. KPIC will allow demonstrating critical component-level and system-level aspects, gain operational experience on segmented telescopes, and enable unique science, vetting the most promising targets for future follow-ups requiring a larger aperture.

\section{ACKNOWLEDGMENTS}

The near-infrared pyramid wavefront sensor is supported by the National Science Foundation under Grant No. AST-1611623. The camera used with the pyramid wavefront sensor is provided by Don Hall with support by the National Science Foundation under Grant No. AST-1106391. The fiber injection unit is supported by the Heising-Simons Foundation. 
The W. M. Keck Observatory is operated as a scientific partnership among the California Institute of Technology, the University of California, and the National Aeronautics and Space Administration. The Observatory was made possible by the generous financial support of the W. M. Keck Foundation. The authors wish to recognize and acknowledge the very significant cultural role and reverence that the summit of Maunakea has always had within the indigenous Hawaiian community. We are most fortunate to have the opportunity to conduct observations from this mountain.

This work was partially carried out at the Jet Propulsion Laboratory, California Institute of Technology, under contract with the National Aeronautics and Space Administration.

\section{REFERENCES}

[1] Mawet, D., Riaud, P., Absil, O., and Surdej, J., "Annular Groove Phase Mask Coronagraph," ApJ 633, 1191-1200 (Nov. 2005).

[2] Delacroix, C., Absil, O., Forsberg, P., Mawet, D., Christiaens, V., Karlsson, M., Boccaletti, A., Baudoz, P., Kuittinen, M., Vartiainen, I., Surdej, J., and Habraken, S., "Laboratory demonstration of a mid-infrared AGPM vector vortex coronagraph," A\&\&A 553, A98 (May 2013).

[3] Vargas Catalán, E., Huby, E., Forsberg, P., Jolivet, A., Baudoz, P., Carlomagno, B., Delacroix, C., Habraken, S., Mawet, D., Surdej, J., Absil, O., and Karlsson, M., "Optimizing the subwavelength grating of L-band annular groove phase masks for high coronagraphic performance," 595, A127 (Nov. 2016).

[4] Huby, E., Bottom, M., Femenia, B., Ngo, H., Mawet, D., Serabyn, E., and Absil, O., "On-sky performance of the QACITS pointing control technique with the Keck/NIRC2 vortex coronagraph," 600, A46 (Apr. 2017).

[5] Serabyn, E., Huby, E., Matthews, K., Mawet, D., Absil, O., Femenia, B., Wizinowich, P., Karlsson, M., Bottom, M., Campbell, R., Carlomagno, B., Defrère, D., Delacroix, C., Forsberg, P., Gomez Gonzalez, C., Habraken, S., Jolivet, A., Liewer, K., Lilley, S., Piron, P., Reggiani, M., Surdej, J., Tran, H., Vargas Catalán, E., and Wertz, O., "The W. M. Keck Observatory Infrared Vortex Coronagraph and a First Image of HIP 79124 B," 153, 43 (Jan. 2017).

[6] Reggiani, M., Christiaens, V., Absil, O., Mawet, D., Huby, E., Choquet, E., Gomez Gonzalez, C. A., Ruane, G., Femenia, B., Serabyn, E., Matthews, K., Barraza, M., Carlomagno, B., Defrère, D., Delacroix, C., Habraken, S., Jolivet, A., Karlsson, M., Orban de Xivry, G., Piron, P., Surdej, J., Vargas Catalan, E., and Wertz, O., "Discovery of a point-like source and a third spiral arm in the transition disk around the Herbig Ae star MWC 758," 611, A74 (Mar. 2018).

[7] Guidi, G., Ruane, G., Williams, J. P., Mawet, D., Testi, L., Zurlo, A., Absil, O., Bottom, M., Choquet, É., Christiaens, V., Castellá, B. F., Huby, E., Isella, A., Kastner, J., Meshkat, T., Reggiani, M., Riggs, A., Serabyn, E., and Wallack, N., "High-contrast imaging of HD 163296 with the Keck/NIRC2 L\#900-band vortex coronograph," (June 2018).

[8] Mawet, D., Choquet, É., Absil, O., Huby, E., Bottom, M., Serabyn, E., Femenia, B., Lebreton, J., Matthews, K., Gomez Gonzalez, C. A., Wertz, O., Carlomagno, B., Christiaens, V., Defrère, D., Delacroix, C., Forsberg, P., Habraken, S., Jolivet, A., Karlsson, M., Milli, J., Pinte, C., Piron, P., Reggiani, M., Surdej, J., and Vargas Catalan, E., "Characterization of the Inner Disk around HD 141569 A from Keck/NIRC2 L-Band Vortex Coronagraphy," 153, 44 (Jan. 2017).

[9] Ruane, G., Mawet, D., Kastner, J., Meshkat, T., Bottom, M., Femenía Castellá, B., Absil, O., Gomez Gonzalez, C., Huby, E., Zhu, Z., Jenson-Clem, R., Choquet, É., and Serabyn, E., "Deep Imaging Search for Planets Forming in the TW Hya Protoplanetary Disk with the Keck/NIRC2 Vortex Coronagraph," 154, 73 (Aug. 2017).

[10] Kirkpatrick, J. D., Gelino, C. R., Cushing, M. C., Mace, G. N., Griffith, R. L., Skrutskie, M. F., Marsh, K. A., Wright, E. L., Eisenhardt, P. R., McLean, I. S., Mainzer, A. K., Burgasser, A. J., Tinney, C. G., Parker, S., and Salter, G., "Further Defining Spectral Type "Y" and Exploring the Low-mass End of the Field Brown Dwarf Mass Function," ApJ 753, 156 (July 2012).

[11] Johnson, J. A., Aller, K. M., Howard, A. W., and Crepp, J. R., "Giant Planet Occurrence in the Stellar Mass-Metallicity Plane," PASP 122, 905-915 (Aug. 2010). 
[12] Swift, J. J., Johnson, J. A., Morton, T. D., Crepp, J. R., Montet, B. T., Fabrycky, D. C., and Muirhead, P. S., "Characterizing the Cool KOIs. IV. Kepler-32 as a Prototype for the Formation of Compact Planetary Systems throughout the Galaxy," ApJ 764, 105 (Feb. 2013).

[13] Dressing, C. D. and Charbonneau, D., "The Occurrence Rate of Small Planets around Small Stars," ApJ 767, 95 (Apr. 2013).

[14] Petigura, E. A., Howard, A. W., and Marcy, G. W., "Prevalence of Earth-size planets orbiting Sun-like stars," Proceedings of the National Academy of Science 110, 19273-19278 (Nov. 2013).

[15] Bowler, B. P., Liu, M. C., Shkolnik, E. L., and Tamura, M., "Planets around Low-mass Stars (PALMS). IV. The Outer Architecture of M Dwarf Planetary Systems," ApJS 216, 7 (Jan. 2015).

[16] Cassan, A., Kubas, D., Beaulieu, J.-P., Dominik, M., Horne, K., Greenhill, J., Wambsganss, J., Menzies, J., Williams, A., Jørgensen, U. G., Udalski, A., Bennett, D. P., Albrow, M. D., Batista, V., Brillant, S., Caldwell, J. A. R., Cole, A., Coutures, C., Cook, K. H., Dieters, S., Prester, D. D., Donatowicz, J., Fouqué, P., Hill, K., Kains, N., Kane, S., Marquette, J.-B., Martin, R., Pollard, K. R., Sahu, K. C., Vinter, C., Warren, D., Watson, B., Zub, M., Sumi, T., Szymański, M. K., Kubiak, M., Poleski, R., Soszynski, I., Ulaczyk, K., Pietrzyński, G., and Wyrzykowski, Ł., "One or more bound planets per Milky Way star from microlensing observations," Nature 481, 167-169 (Jan. 2012).

[17] Benneke, B., "Strict Upper Limits on the Carbon-to-Oxygen Ratios of Eight Hot Jupiters from SelfConsistent Atmospheric Retrieval," arXiv.org, 7655 (Apr. 2015).

[18] Crossfield, I. J. M., Biller, B., Schlieder, J. E., Deacon, N. R., Bonnefoy, M., Homeier, D., Allard, F., Buenzli, E., Henning, T., Brandner, W., Goldman, B., and Kopytova, T., "A global cloud map of the nearest known brown dwarf," Nature 505, 654-656 (Jan. 2014).

[19] Snellen, I. A. G., Brandl, B. R., de Kok, R. J., Brogi, M., Birkby, J., and Schwarz, H., "Fast spin of the young extrasolar planet $\beta$ Pictoris b," Nature 509, 63-65 (May 2014).

[20] Snellen, I., de Kok, R., Birkby, J. L., Brandl, B., Brogi, M., Keller, C., Kenworthy, M., Schwarz, H., and Stuik, R., "Combining high-dispersion spectroscopy with high contrast imaging: Probing rocky planets around our nearest neighbors," A $\mathscr{E} A$ 576, A59 (Apr. 2015).

[21] Costa, J. B., Hippler, S., Feldt, M., Esposito, S., Ragazzoni, R., Bizenberger, P., Puga, E., and Henning, T. F. E., "PYRAMIR: a near-infrared pyramid wavefront sensor for the Calar Alto adaptive optics system," in [Adaptive Optical System Technologies II], Wizinowich, P. L. and Bonaccini, D., eds., Proc. SPIE 4839, 280-287 (Feb. 2003).

[22] Furlan, E., Luhman, K. L., Espaillat, C., D’Alessio, P., Adame, L., Manoj, P., Kim, K. H., Watson, D. M., Forrest, W. J., McClure, M. K., Calvet, N., Sargent, B. A., Green, J. D., and Fischer, W. J., "The Spitzer Infrared Spectrograph Survey of T Tauri Stars in Taurus," ApJS 195, 3 (July 2011).

[23] Mawet, D., Ruane, G., Xuan, W., Echeverri, D., Klimovich, N., Randolph, M., Fucik, J., Wallace, J. K., Wang, J., Vasisht, G., Dekany, R., Mennesson, B., Choquet, E., Delorme, J.-R., and Serabyn, E., "Observing Exoplanets with High-dispersion Coronagraphy. II. Demonstration of an Active Single-mode Fiber Injection Unit," 838, 92 (Apr. 2017).

[24] Wang, J., Mawet, D., Ruane, G., Hu, R., and Benneke, B., "Observing Exoplanets with High Dispersion Coronagraphy. I. The Scientific Potential of Current and Next-generation Large Ground and Space Telescopes," 153, 183 (Apr. 2017).

[25] Snellen, I. A. G., de Kok, R. J., de Mooij, E. J. W., and Albrecht, S., "The orbital motion, absolute mass and high-altitude winds of exoplanet HD209458b," 465, 1049-1051 (June 2010).

[26] Birkby, J. L., de Kok, R. J., Brogi, M., de Mooij, E. J. W., Schwarz, H., Albrecht, S., and Snellen, I. A. G., "Detection of water absorption in the day side atmosphere of HD $189733 \mathrm{~b}$ using ground-based high-resolution spectroscopy at $3.2 \mu \mathrm{m}, "$ 436, L35-L39 (Nov. 2013).

[27] de Kok, R. J., Birkby, J., Brogi, M., Schwarz, H., Albrecht, S., de Mooij, E. J. W., and Snellen, I. A. G., "Identifying new opportunities for exoplanet characterisation at high spectral resolution," 561, A150 (Jan. 2014).

[28] Brogi, M., de Kok, R. J., Birkby, J. L., Schwarz, H., and Snellen, I. A. G., "Carbon monoxide and water vapor in the atmosphere of the non-transiting exoplanet HD 179949 b," 565, A124 (May 2014).

[29] Zhu, Z., "Accreting Circumplanetary Disks: Observational Signatures," 799, 16 (Jan. 2015).

[30] Vanderburg, A., Rappaport, S. A., and Mayo, A. W., "Detecting Exomoons Via Doppler Monitoring of Directly Imaged Exoplanets," ArXiv e-prints (May 2018). 